\title{
Research on the Application of Artificial Intelligence Technology in Human Resource Management
}

\author{
Fengxiang Jiang ${ }^{1}$, Jie $\mathrm{Li}^{2}$, Moutao $\mathrm{Du}^{3}$, Fang Wang ${ }^{4}$ \\ ${ }^{1,2,3,4}$ Xi’an Peihua University, Xi’an Shaanxi, 710125
}

Keywords: Artificial Intelligence Technology; Human Resource Management; Application

\begin{abstract}
Machines have gradually replaced low-skilled, low-wage, and high-repetition jobs since the industrial revolution. Some smart devices were hidden behind the scenes in the past; artificial intelligence is getting more and more attention with the advent of technology. Many workplaces have been reshaped in the era of artificial intelligence, work patterns have been changed, many jobs have been redefined, and so has the work of human resources management. In the future, a lot of work in human resources management may be replaced by artificial intelligence. But what needs to be done in the future is more judgment work than repetitive work. Human resource practitioners will not disappear, but the work content will change from low-value and repetitive work to planning, judging, organizing strategies, and emotional input. This paper mainly studies the influence of artificial intelligence technology on the six modules of human resource management and the concrete application of artificial intelligence technology in human resource management.
\end{abstract}

\section{Introduction}

With the development of artificial intelligence technology, human resource management has been greatly challenged. Artificial intelligence has replaced most of the human repetitive operational work, but some of the work artificial intelligence cannot be completed. Therefore, especially the organization innovation work still needs to attach importance to the human resources management in the artificial intelligence era, and the core of human resources management development is to the understand and respect the human resource. The participation of people cannot solve all problems in a stylized way in an organization. With the development of artificial intelligence to improve human resource management, the part that cannot be replaced by artificial intelligence pays attention to humanistic concern, strengthens organization support, and acquires more organization commitment of human resources. Human resource managers need to change their thinking in the face of the rapid development of artificial intelligence, fully recognize the impact of artificial intelligence on human resources, strive to optimize their own quality, strive to meet the trend of development of the era, and actively stimulate their own creativity and passion, and give play to the uniqueness of human beings.

\section{The influence of artificial intelligence technology on the six modules of human resources}

Artificial intelligence is a kind of machine ability to imitate human ability, such as learning, problem-solving and perceptual ability. Artificial intelligence has profound influence on human resource management. This paper mainly analyzes the influence of artificial intelligence technology on six modules of human resources.

\subsection{Human resources planning}

Human resources management activities of enterprises can be more forward-looking and leading through human resource planning, and the best allocation of human resources and other resources can be realized. Artificial intelligence has built a good digital infrastructure for human resources planning. The human resource planning needs to make the demand and supply forecast, make the plan to balance the supply and demand, according to the model and the algorithm can improve the accuracy of the plan, ensure that all kinds of jobs in the enterprise can get the right people at the 
right time, including the quantity, quality, hierarchy and structure of human resources, the effective realization of their potential, and specific programs based on projected results, including recruitment, dismissal, promotion, training, wage and welfare policies, Echelon construction and organizational change.

\subsection{Human resources recruitment}

The influence of artificial intelligence has been seen in the field of recruitment, where a particular phase of the recruitment journey promoted by artificial intelligence is video interviews, the use of chat robots to deliver personalized experiences online and to simplify part of the recruitment cycle. Hiring teams are overburdened with administrative tasks, such as screening resumes and conducting initial interview screens. However, as organizations adopt artificial intelligence recruitment tools, such as text-based interview and automated planning solutions, these tedious management tasks will be eliminated, making recruiters time more strategic and focused on engagement, to interview and recruit suitable talent. Artificial intelligence and robotics have opened up exciting new functions for human resources. Software now recognizes faces and gender, identification, image recognition, listening to sounds and emotions, and decoding video interviews to identify educational levels, lies, and cognitive abilities. Analytical tools can intelligently select candidates, determine employee career choices, and guide managers to improve leadership skills.

\subsection{Human resources training}

Before training, artificial intelligence can find out the shortcomings of human resources in enterprises, and need to take targeted measures in terms of skills, that is, to analyze the human resources training needs of enterprises through artificial intelligence. In training, using artificial intelligence is to effectively analyze the shortage and advantage of employees, to play the role of training, to improve the training effect, to establish a dynamic training curriculum system, the traditional training methods cannot meet the training needs in real time, the replacement of human

costs, Artificial intelligence exerts its intelligence and has higher efficiency; After training, the training effect is evaluated by artificial intelligence.

\subsection{Compensation management}

Enterprises are at different stages and compensation is not the same. The adjustment of salary should have strategic orientation and adapt to the adjustment of enterprise's strategic goal. We can analyze the external and internal equilibrium of the compensation of the enterprise through artificial intelligence, to complete the salary survey and the post evaluation, and design the appropriate salary structure to determine the grade and scope of the compensation. Finally, the salary adjustment policy is formulated.

\subsection{Performance management}

Artificial intelligence can reduce manpower input for daily performance reviews, and the evaluation is more accurate and objective, but it only applies to some types of work, It is impossible for those innovative jobs to simply rely on the daily work of performance management or need to be weighed to determine the value of its work. Artificial intelligence assessment can comprehensively assess the actual performance of employees in the work, help employees to enhance their personal self-awareness, timely and initiative to find the development gap with the team members, help the team to find out the gap, clear the direction of development, and then improve the efficiency of the organization.

\subsection{Labor relations management}

The relationship between artificial intelligence and labor and employment problems is substitution, complementary and creative effects coexisting. Artificial intelligence will replace a lot of simple, repetitive work, which is a challenge to labor relations management, but in labor relations management, the basic work of labor relations, such as employee entry, employee file management, in the aspects of turnover process and company policy explanation, artificial intelligence can be 
used as virtual assistant, which can reduce labor force, reduce communication and work efficiency. It can help enterprises save manpower cost in the long run.

\section{Application of artificial intelligence in human resource management}

Many traditional positions of enterprises are replaced by machines under the background of artificial intelligence technology, the automation level of production and management of enterprises is higher, more new posts appear, the core of competition is knowledge, and the repetitive work has been greatly optimized. Artificial intelligence raises the production efficiency of the enterprise, improves the management efficiency, and stimulates the enthusiasm and creativity of the staff. At present, many industries have been deeply affected by artificial intelligence, and countless scenarios have been changed by artificial intelligence, including manufacturing, service, and even marketing, retail, and logistics and so on. In addition to continuously improving the quality of products and services and optimizing the rapid iteration of products, enterprises should integrate the artificial intelligence thinking with the concept of talent management in order to make the talents become the soft power of the enterprises.

There may be more unattended supermarkets in the future. Yun Ma and Wahaha Group Chairman, Qinghou Zong, have announced that there will be 100,000 unattended supermarkets across the country in the next few years. JingDong CEO, Qiangdong Liu, also announced the opening of 500,000 convenience stores across the country, as well as a large number of unattended supermarkets. What does the appearance of unattended supermarket mean? Big data and artificial intelligence sneaks into our life, silently replaced many posts, so that the employment environment has become more severe.

\section{The application and innovation of artificial intelligence technology in human resource management}

Artificial intelligence is the ability of machines to imitate human capabilities, such as learning, problem-solving, and perception. For HR, it is a functional application of artificial intelligence that simplifies and automates some workflows. Therefore, the human resources management of enterprise needs to effectively apply artificial intelligence technology.

\subsection{To restructure the human resources management model}

Human resources work should focus on staff experience, humanistic care, and let innovation become the core strategy of human resources department in the era of artificial intelligence. Human resources needs to redefine itself as a team that helps managers and employees transform quickly and adapt to new thinking patterns. It is important to actively apply artificial intelligence, let artificial intelligence for human resources management services. We actively seek the joint position and cross the gap of artificial intelligence actively in the business. At the same time, artificial intelligence issued the correct instructions through the transformation of thinking, which means artificial intelligence to guide the correct direction of development.

\subsection{To reposition human resources management}

Human resources do more judgment than repetitive work in the era of artificial intelligence. We must find the correct location, learn to dig out the key ability of human's non-logical elements, and fit the deviation between human and artificial intelligence. Go ahead of artificial intelligence, strive to be free from routine work, focus on core functions, and focus on creating more value for employees. Human resource management will be transformed from a real employee to a conceptual virtual labor force. We need to make full use of artificial intelligence, and the overall workforce to adapt to the latest needs of the new era. We should learn to use the integrated cloud platform instead of the traditional system, so as to build a good digital infrastructure of human resources management, develop long-term human resources technology strategy, and set up a human resources digital team. 


\subsection{To use artificial intelligence to help recruit, manage and optimize staff}

The principles of artificial intelligence for staff management are similar. Artificial intelligence will enable companies to make better hiring decisions. Artificial intelligence will enable businesses to manage their teams more effectively. One of the biggest advantages of artificial intelligence is its ability to screen out millions of unstructured data points and understand them quickly. Companies can use this feature to mine social and behavioral data and to better match the right person for the position. Using machine learning prediction analysis to improve efficiency, reduce operating costs, increase income and improve the quality of talent provides the possibility.

\subsection{To improve working environment using artificial intelligence}

It is necessary to use artificial intelligence to improve the work environment, enhance the interactive experience in the work environment. Artificial intelligence can also improve the various experiences of employee; it is to realize the hypothetical situation with the help of augmented reality. Employees can watch videos and even have two-way conversations with company manuals with a simple phone call. With the increase of managers and employees, it is necessary to have the ability to make use of augmented reality to make the company's physical position or workspace a reality has great potential to improve work efficiency.

\section{Conclusion}

All in all, artificial intelligence has gradually penetrated into people's daily life and work. Artificial intelligence will have big influences on some professions in the future, but it will also promote some career development and bring new employment opportunities. But in practice, people have found that it is not just entry-level occupations that are replaced by artificial intelligence. Some middle-level and even higher-level occupations are threatened, more subversive than the occupation development in the previous industrial revolution. The amalgamation of artificial intelligence and existing technology has solved a large number of problems for enterprises. Enterprises need more new ideas and ways to use artificial intelligence skills to manage human resources in the future.

\section{References}

[1] Yinlong Li, Xiaohong Wu. On The Influence of Artificial Intelligence on Human Resource Management [J]. Human Resources Management, 2018 (3): 400-401.

[2] Liang Wang. Using Artificial Intelligence Technology to Improve the Level of Human Resources Management [J]. Sinopec, 2017 (7): 53-54.

[3] Jing Yun. Reflections on Human Resource Management Brought by Digitization [J]. China Telecom, 2018 (2): 72-74

[4] Yuankun Hong. Reflections on the Reform of Enterprise Human Resource Management in Big Data Era [J]. Human Resources Management, 2018 (3): 3-3.

[5] Chenchen Liu. Reflections on the Reform of Enterprise Human Resource Management in Big Data Era [J]. Development of Human Resources, 2016 (8): 143-143.

[6] Ruigong Wen, Yujie Wang. Analysis of Human Resource Management Reform in the Era of Big Data [J]. Modern Economic Information, 2017 (2): 139-139. 